\title{
LEPTINEMIA DE JEJUM EM CRIANÇAS E ADOLESCENTES EUTRÓFICOS
}

Carlos Alberto Nogueira de Almeida ${ }^{1 *}$, Adriana Pelegrino Pinho Ramos², Iguatemy Lourenço Brunetti ${ }^{3}$, Maria Teresa Pepato ${ }^{4}$, Rubens Garcia Ricco ${ }^{5}$

Trabalho realizado no Centro de Estudos em Saúde e Nutrição Infanto-Juvenil (CESNI) da Universidade de Ribeirão Preto, S. Paulo, SP

*Correspondência:

Avenida Portugal, $\mathrm{n}^{\circ} 1620$

- Apto. 73

CEP: $14020-380$

Ribeirão Preto - SP

\section{RESUMO}

OвJetivo. Determinar, em um grupo de crianças e adolescentes eutróficos, os valores de leptinemia de jejum e sua correlação com idade e Índice de Massa Corporal (IMC).

Métodos. Estudo de corte transversal realizado em duas escolas públicas de Ribeirão Preto, Brasil. Foram obtidos medidas antropométricas e amostra de sangue venoso em jejum para dosagem da leptinemia de 448 crianças e adolescentes eutróficos, maturadores médios, de ambos os sexos, com idades entre 7 e 17,9 anos. Utilizando-se o teste de Mann-Whitney foram realizadas comparações entre as concentrações obtidas para meninos e meninas em cada faixa etária. Posteriormente, utilizandose o teste de Kruskal-Wallis, foram comparados os valores em cada faixa etária e, por meio do teste de correlação de Spearman, foi avaliada a correlação entre a leptinemia de jejum e a idade e entre leptinemia de jejum e o escore z do IMC.

Resultados. O valores de leptinemia de jejum diferiram entre meninos e meninas em todas as faixas etárias sendo sempre mais elevados para as meninas. Entre os meninos, os valores de leptinemia não apresentam variação estatisticamente significativa; entre as meninas, observou-se variabilidade, com aumento progressivo de acordo com a faixa etária. O estudo de correlação, mostrou que a leptinemia apresenta correlação positiva com o escore z do índice de massa corporal em ambos os sexos. Em relação à idade, observou-se correlação apenas entre as meninas.

Conclusão. Os dados apontam para a necessidade do estabelecimento de curvas de referência para a leptinemia de jejum que levem em conta o sexo, a idade e o IMC.

Unitermos: Leptina. Índice de Massa Corporal. Tecido adiposo. Criança. Adolescente. Valores de referência.

\section{INTRODUÇÃo}

A leptina é um hormônio codificado pelo gene ob presente nos adipócitos que, por meio de sinalização ao sistema nervoso central, reduz o consumo cumulativo de alimentos por meio da supressão do apetite, promovendo a utilização das reservas de gordura ${ }^{1}$ e controlando a homeostase energética ${ }^{2}$. Exerce efeitos em vários órgãos, nos sistemas reprodutor, renal e cardiovascular e apresenta, ainda, um papel metabólico, pois estimula a gliconeogênese e inibe a glicogenólise ${ }^{3,4}$, além de facilitar 0 consumo de glicose e de melhorar a sensibilidade à insulina(4).

Dentro do processo de maturação que caracteriza a adolescência, tem sido evidenciado que a leptina apresenta importante papel como sinalizadora do momento de início da puberdade ${ }^{5}$ cujo mecanismo seria, pelo menos entre as meninas, de origem evolutiva, uma vez que se espera um percentual mínimo de gordura para que se possa assegurar uma gestação futura ${ }^{6}$. Dessa forma, a sinalização da massa adiposa efetuada pela leptina teria, também, a função de informar ao sistema nervoso central que o percentual mínimo de gordura foi atingido e que a puberdade pode começar?.

Níveis plasmáticos elevados de leptina estão associados a diversos fatores relacionados à síndrome metabólica ${ }^{8}$ como: - IMC, a insulinemia de jejum, a sensibilidade à insulina, a trigliceridemia, a pressão arterial e a circunferência abdominal ${ }^{9}$. Dessa forma, acredita-se que a resistência à leptina também possa ser um componente importante da síndrome metabólica ${ }^{10}$ e um provável marcador de risco de doença coronariana ${ }^{11}$. Na infância, o aumento nos níveis de leptina é característica comum da obesidade. ${ }^{12}$.

Independentemente da composição corporal inicial, valores aumentados de leptina predispõe ao aumento progressivo do IMC e da porcentagem de gordura em crianças $^{13}$. Entretanto, uma das maiores dificuldades, tanto para estudos científicos quanto para a clínica médica, é encontrar os adequados pontos de corte para a avaliação da leptinemia. No sentido de contribuir com essa questão, o presente estudo procurou avaliar, em um grupo de crianças e adolescentes eutróficos, os valores das

\footnotetext{
1. Professor titular do Curso de Medicina da Universidade de Ribeirão Preto e Coordenador do CESNI, Ribeirão Preto, SP

2. Coordenadora do Laboratório de Análises Clínicas da Universidade de Ribeirão Preto, Ribeirão Preto, SP

3. Professor assistente doutor - Diretor da Faculdade de Ciências Farmacêuticas de Araraquara - UNESP, Araraquara, SP

4. Professora da Faculdade de Ciências Farmacêuticas de Araraquara - UNESP, Araraquara, SP

5. Professor associado da Faculdade de Medicina de Ribeirão Preto da USP, Ribeirão Preto, SP
} 
concentrações de leptinemia de jejum e sua correlação com o sexo, a idade e o IMC.

\section{Métodos}

O estudo foi realizado nas duas únicas escolas públicas do distrito de Bonfim Paulista, localizado a cerca de seis quilômetros de Ribeirão Preto (sudeste do Brasil) e administrativamente subordinado a essa cidade. Foi desenhado como um estudo de corte transversal em que, em um mesmo momento, foram obtidas medidas antropométricas e colhidas amostras de sangue venoso de 448 crianças e adolescentes eutróficos, de ambos os sexos, com idades entre 7 e 17,9 anos. Para se evitar que dados provenientes de adolescentes com maturação tardia ou precoce pudessem produzir erros na avaliação, foram considerados para o estudo somente aqueles que se encontravam dentro do estadiamento esperado para sua idade, segundo critérios de Tanner ${ }^{14}$.

0 estadiamento puberal foi avaliado utilizando-se figuras representativas dos diferentes estágios, sendo solicitado ao participante que apontasse as figuras que melhor refletissem seu desenvolvimento. Para evitar constrangimento e garantir a consistência dos dados, essa avaliação foi sempre realizada por profissional do mesmo sexo que o entrevistado durante 0 procedimento de antropometria, sendo um médico pediatra para os meninos e uma enfermeira padrão para as meninas. Esses dois profissionais já atuavam nas escolas há bastante tempo, em atividade docente-assistencial, o que permitia uma relação de confiança mútua entre entrevistadores e entrevistados. Em todos os casos em que restou dúvida quanto à resposta, foi feita reavaliação, por um dos dois profissionais citados, por meio de exame físico. A opção pela autoavaliação do estadiamento puberal foi feita devido à dificuldade de se realizar exame clínico de genitais em um ambiente escolar, o que poderia provocar menor adesão dos adolescentes ao estudo. Sendo assim, inicialmente todos os 1200 estudantes matriculados nas escolas foram considerados para a inclusão no estudo, tendo-se como critério de exclusão aqueles que: apresentavam percentil de IMC abaixo de 5 ou acima de 85 ( $n=187)$, não concordaram em participar $(n=218)$, não apresentaram o termo de consentimento assinado pelos responsáveis $(n=305)$, apresentavam estadiamento puberal incompatível com a idade $(n=22)$, apresentavam qualquer doença ativa em tratamento $(n=18)$ ou apresentavam condições inadequadas para antropometria como uso de próteses, gesso, deficiências físicas, etc $(n=2)$, chegando-se ao número final de 448 participantes.

Antes da coleta de materiais biológicos, os participantes e seus responsáveis foram esclarecidos a respeito do projeto verbalmente e por meio do termo de consentimento livre e esclarecido, tendo-se obtido autorização para a participação. Todos foram orientados a fazer jejum de 12 horas, realizando-se antropometria e coleta de sangue pela manhã nas escolas estaduais participantes do estudo. As aferições de peso e estatura seguiram as recomendações da Organização Mundial de Saúde ${ }^{15}$. De cada sujeito, foram coletados $4 \mathrm{~mL}$ de sangue em frasco sem aditivo e encaminhado em até duas horas ao laboratório para o processamento da amostra e a realização da análise hormonal. 0 transporte foi realizado em caixas térmicas refrigeradas e 0 material biológico separado em centrífuga Bio Eng modelo BE 4000 por cinco minutos a 3.500 rpm entre uma e duas horas e meia após a coleta (tempo suficiente para a coagulação do sangue). Após a centrifugação, o soro foi separado e foi realizado ensaio imunoenzimático tipo sanduíche com resultado em leitora de ELISA Organon para dosagem da leptinemia.

Com finalidade de avaliar a hipótese da existência de variação nos valores do indicador estudado de acordo com a idade e o gênero e, ao mesmo tempo, garantir um número adequado de indivíduos em cada grupo, optou-se por dividir os 448 participantes da seguinte maneira: meninos e meninas e cinco faixas de idade. Todas as faixas etárias obedeceram a intervalo de dois anos, à exceção da última (15 a 17,9 anos) que teve intervalo de três anos. Com esses critérios, foi possível estudar grupos sempre superiores a 25 indivíduos, o que se mostrou adequado durante a análise estatística. Utilizando-se o teste de Mann-Whitney foram realizadas comparações entre os valores obtidos para meninos e meninas em cada faixa etária. Posteriormente, utilizando-se o teste de Kruskal-Wallis, foram comparados os valores em cada faixa etária para meninos e meninas. Também foi avaliada, pelo teste de correlação de Spearman, a correlação entre o indicador leptinemia de jejum e a idade e entre leptinemia de jejum e o escore z do IMC, separadamente para meninas e meninos.

O trabalho foi aprovado pelo comitê de ética da Universidade de Ribeirão Preto em 20 de outubro de 2003, conforme consta no parecer do memorando ComÉt/n o 94/2003.

\section{Resultados}

Os resultados obtidos estão apresentados nas Tabelas 1 e 2 .

0 valores de leptinemia de jejum diferem entre meninos e meninas em todas as faixas etárias estudadas e os valores crescentes das diferenças entre as médias apontam para um incremento dessa diferença com o passar da idade. Observase, também, que as concentrações de leptina são sempre mais elevadas para as meninas.

Em relação à evolução por faixa etária, nos meninos, os valores de leptinemia não apresentam variação estatisticamente significativa; contudo, nas meninas, aparece variabilidade com aumento progressivo de acordo com a faixa etária.

O estudo de correlação mostrou que a leptinemia apresenta correlação positiva com o escore z do IMC em ambos os sexos, podendo ser considerada fraca entre meninos e moderada entre meninas. Em relação à idade, não se observou correlação entre meninos, mas, entre as meninas, a correlação mostrou-se positiva e de moderada intensidade.

\section{Discussão}

Apesar de ser atualmente reconhecido o fato de que indivíduos obesos apresentem valores elevados de leptina circulante, tem crescido o interesse científico para se conhecer como esse hormônio se comporta entre os eutróficos. Isso se deve ao fato de que, aparentemente, valores elevados determinam maior risco de resistência insulínica ${ }^{16}$ e obesidade futura ${ }^{13,17,18}$, o que pode ser clinicamente bastante útil sob o aspecto preventivo, especialmente em famílias geneticamente predispostas à obesidade.

Wallace et al. demonstraram, em extenso estudo prospectivo, que a leptinemia é um fator de risco independente para doença coronariana $^{19}$, o que tem sido confirmado em outros estudos recentes $^{11,20}$. Mesmo entre crianças e adolescentes obesos tem 
Tabela 1 - Média e desvio padrão dos valores de leptinemia de jejum de meninos e meninas eutróficos de acordo com a faixa etária

\begin{tabular}{|c|c|c|c|c|c|}
\hline \multirow{2}{*}{ Idade } & \multicolumn{5}{|c|}{ leptinemia de jejum (ng/dL) } \\
\hline & \multicolumn{2}{|c|}{ Meninas } & \multicolumn{2}{|c|}{ Meninos } & $p^{* *}$ \\
\hline 7 a 8,9 anos & 38 & $3,67(2,61)$ & 30 & $2,53(2,66)$ & 0,0347 \\
\hline 11 a 12,9 anos & 60 & $11,23(7,62)$ & 37 & $3,84(5,63)$ & $<0,0001$ \\
\hline 13 a 14,9 anos & 69 & $16,41(11,88)$ & 58 & $4,04(6,59)$ & $<0,0001$ \\
\hline 15 a 17,9 anos & 48 & $20,39(10,46)$ & 26 & $2,97(4,50)$ & $<0,0001$ \\
\hline
\end{tabular}

* Teste de Kruskal-Wallis para comparação simultânea de mais de dois grupos

** Teste de Mann-Whitney

Tabela 2 - Correlação entre leptinemia de jejum e idade e leptinemia de jejum e escore z de IMC entre meninos e meninas eutróficos

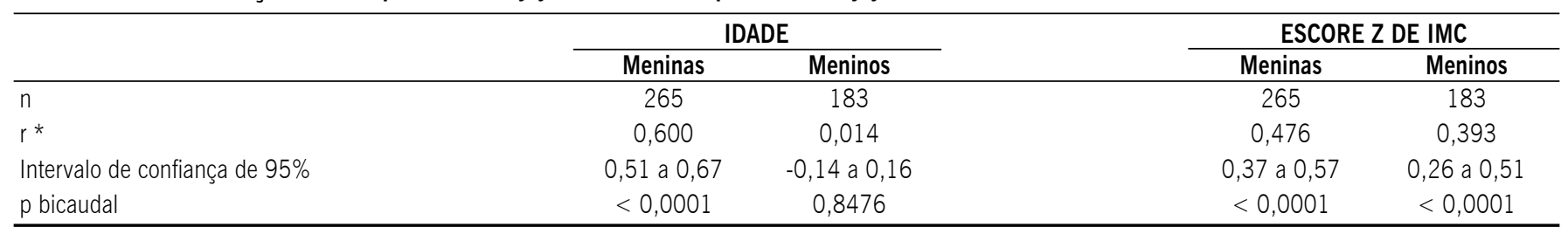

* Coeficiente de correlação de Spearman

sido demonstrado que nem todos apresentam-se hiperleptinêmicos e, entre aqueles que o são, é comum a associação com outros fatores de risco, como hiperinsulinismo, dislipidemia e hipertensão arterial $^{10}$.

Para se estabelecer intervalos de referência para parâmetros biológicos, é fundamental que a investigação seja realizada obtendo-se valores que impliquem em risco associado. Entretanto, o desenho de estudos para cumprir esse quesito exige longo tempo de observação e, frequentemente, é preciso recorrer, mesmo que provisoriamente, a estudos de corte transversal para se observar a variação estatística desse parâmetro. 0 presente estudo buscou descrever, por meio de avaliação transversal com crianças e adolescentes eutróficos de diferentes faixas etárias, o comportamento da leptinemia de jejum. Pôde-se observar, primeiramente, que existe diferença entre os sexos, de modo que uma eventual curva de referência deverá, necessariamente, ser sexo-específica. Essa diferença para leptinemia também têm sido descrita em outros estudos: Poveda et al. ${ }^{21}$, em estudo que avaliou transversalmente 545 crianças colombianas de 5 a 15 anos e Garcia-Mayor et al. ${ }^{7}$, avaliando 789 crianças espanholas na mesma faixa etária, verificaram que havia diferença entre os sexos e, de modo semelhante ao presente estudo, que as meninas apresentavam valores mais elevados.

Também se verificou que, entre os meninos, a leptinemia não apresentou variação significativa com as diferentes faixas etárias, ao contrário das meninas em que os valores tendem a crescer. Esse resultado difere ligeiramente em relação a outros estudos no que diz respeito aos meninos. Em estudo longitudinal, que acompanhou 40 crianças dos 8 aos 16 anos, Ahmed et al. ${ }^{18}$ verificaram que a leptinemia aumentava com a idade entre as meninas, mas, entre os meninos, aumentava até o início da puberdade e, após esse período, passava a apresentar redução. Outros estudos também demonstram essa tendência7;21. No presente estudo, de fato os dados mostram tendência semelhante, com valores de leptina entre meninos apresentando valores máximos na faixa entre 13 e 14,9 anos, caindo em seguida, mas essa dinâmica não se mostrou estatisticamente significativa. Por outro lado, Brandão et al. ${ }^{22}$, avaliando 175 adolescentes saudáveis, com idades entre 10 e 18 anos e não obesos, verificaram correlação negativa entre idade e leptinemia entre os meninos. Essas diferenças entre os estudos refletem basicamente a ausência de padronização metodológica, o que leva a resultados distintos já que a leptinemia sofre influência marcante de indicadores como estadiamento puberal, porcentagem de gordura corporal, entre outros, o que dificulta a comparação entre os estudos. Deve-se enfatizar que, no presente estudo, observou-se dispersão acentuada nos valores de leptinemia, com desvio padrão próximo, ou até superior a média em algumas faixas etárias. Também é fato que, mesmo que a finalidade tenha sido de descrever o comportamento da leptinemia em um grupo com características tão próximas quanto possível da normalidade, tendo-se incluído apenas indivíduos saudáveis, eutróficos e maturadores médios, mesmo entre os maturadores médios é possível que existam diferenças entre o estadiamento puberal que a avaliação por faixa etária possa ter escondido. Por esse motivo, novos estudos podem ser necessários para avaliar a correlação entre a leptinemia e o estadiamento puberal.

Outro aspecto verificado diz respeito à correlação positiva observada, ainda que fraca, para ambos os sexos, entre a leptinemia e o escore z de IMC, mesmo considerando-se que o grupo estudado era de indivíduos eutróficos. Isso significa que, para crianças e adolescentes, mesmo antes de atingir valores indicativos de sobrepeso, apresentar maior massa corporal implica em, também, apresentar concentrações mais elevadas 
de leptina plasmática. Essa observação deve ser levada em conta para o estabelecimento de curvas de referência, já que é bastante provável que, fisiologicamente, sejam esperados valores maiores de leptinemia em indivíduos mais pesados, sem que isso represente qualquer problema. Por outro lado, o presente estudo apresenta a limitação de não ter avaliado a composição corporal dos sujeitos envolvidos, o que pemitiria correlacionar a leptinemia diretamente com a adiposidade.

Outros estudos que avaliaram a correlação entre IMC e leptinemia, independentemente da inclusão ${ }^{7}$ ou exclusão ${ }^{22}$ de indivíduos obesos na análise, também demonstraram correlação positiva entre esses indicadores. Esse resultado corrobora com o modelo da leptina, um dos principais sinalizadores para o início da puberdade ${ }^{5}$. De fato, a observação clínica tem demonstrado de forma inequívoca que crianças obesas tendem a iniciar mais precocemente a puberdade ${ }^{6}$, mas pouco tem se estudado essa correlação entre as eutróficas. No presente estudo, foram envolvidas apenas as maturadoras médias, ou seja, aquelas em que os eventos puberais estavam compatíveis com o esperado para as diferentes faixas de idade cronológica. Mesmo assim, a leptinemia não se apresentou constante, o que pode coadunar com a grande variabilidade biológica, em relação ao início e à duração da puberdade, já exaustivamente descrita entre os indivíduos normais, indicando que a massa corporal deva contribuir com essa variabilidade.

Os dados do presente estudo apontam para a necessidade da construção de curvas de referência para a leptinemia de jejum que respeitem, pelo menos, para os meninos, o IMC e, para as meninas, o IMC e a faixa etária. A evolução do conhecimento sobre a leptina tem demosntrado, cada vez mais, a importância de se classificar os indivíduos como portadores ou não de hiperleptinemia. Por outro lado, a variação apresentada na concentração desse hormônio em relação a variáveis estudadas nesse e em outros estudos, como sexo, idade, estadiamento puberal, adiposidade, etc, faz com que essa definição passe, necessariamente, na faixa etária pediátrica, pela avaliação por meio de curvas que representem as faixas de variação fisiológicas e as distingam das situações de risco.

\section{Suporte FinANCEIRO:}

Universidade de Ribeirão Preto e Faculdade de Ciências Farmacêuticas da UNESP de Araraquara

\section{Conflito de interesse: não há}

\section{SUMmARY}

\section{Fasting LePTINEMIA IN EUTROPHIC CHILDREN AND ADOLESCENTS}

OBJECTIVE. To determine, in a group of eutrophic children and adolescents, the values of fasting leptinemia and its correlation with age and body mass index.

Methods. A cross-sectional study conducted in two public schools in Ribeirao Preto, Brazil. Anthropometric measurements and venous blood samples were obtained for determination of fasting leptinemia of 448 eutrophic and medium maturers children and adolescents, of both genders, aged between 7 and 17.9 years. Using the Mann-Whitney test, comparisons were made between the concentrations obtained for boys and girls in each age group. Subsequently, using the Kruskal-Wallis test, values were compared in each age group and, using the Spearman correlation test, the correlations between fasting leptinemia and age and between fasting leptinemia and the z-scores of body mass index were assessed.

RESULTS. Fasting leptinemia values differed between boys and girls in all age groups and it was higher for girls. Among boys, the values of leptinemia did not show statistically significant variation; among girls, there was variability, with gradual increase according to age group. The correlation study showed positive correlation between leptinemia and z-scores of body mass index in both genders and between leptinemia and age only for girls.

CONCLUSION. The data show the necessity of establishing reference curves for fasting leptinemia taking into account gender age and body mass index. [Rev Assoc Med Bras 2009; 55(4): 463-7]

Key words: Leptin. Body Mass Index. Adipose tissue. Child. Adolescent. Reference values.

\section{REFERÊNCIAS}

1. Halaas JL, Friedman JM. Leptin and its receptor. J Endocrinol. 1997; 155(2):215-6.

2. Gao Q, Horvath TL. Cross-talk between estrogen and leptin signaling in the hypothalamus. Am J Physiol Endocrinol Metab. 2008;294(5):E817-E26.

3. Friedman JM. The function of leptin in nutrition, weight, and physiology. Nutr Rev. 2002;60(10 Pt 2):S1-14.

4. Havel PJ. Role of adipose tissue in body-weight regulation: mechanisms regulating leptin production and energy balance. Proc Nutr Soc. 2000;59(3):359-71

5. Clayton PE, Trueman JA. Leptin and puberty. Arch Dis Child. 2000;83(1):1-4.

6. Kaplowitz PB. Link between body fat and the timing of puberty. Pediatric. 2008;121(Suppl 3):S208-S17.

7. Garcia-Mayor RV, Andrade MA, Rios M, Lage M, Dieguez C, Casanueva FF. Serum leptin levels in normal children: relationship to age, gender, body mass index, pituitary-gonadal hormones, and pubertal stage. J Clin Endocrinol Metab. 1997;82(9):2849-55.

8. Haffner SM, Mykkanen L, Rainwater DL, Karhapaa P, Laakso M. Is leptin concentration associated with the insulin resistance syndrome in nondiabetic men? Obes Res. 1999;7(2):164-9.

9. De Almeida CA, Pinho AP, Ricco RG, Elias CP. Abdominal circumference as an indicator of clinical and laboratory parameters associated with obesity in children and adolescents: comparison between two reference tables. J Pediatr (Rio J). 2007;83(2):181-5.

10. Valle M, Gascon F, Martos R, Bermudo F, Ceballos P, Suanes A. Relationship between high plasma leptin concentrations and metabolic syndrome in obese pre-pubertal children. Int J Obes Relat Metab Disord. 2003;27(1):13-8.

11. Dubey L, Zeng HS, Wang HJ, Liu RY. Potential role of adipocytokine leptin in acute coronary syndrome. Asian Cardiovasc Thorac Ann. 2008;16(2):124-8.

12. Caprio S, Tamborlane WV, Silver D, Robinson C, Leibel R, McCarthy S, et al. Hyperleptinemia: an early sign of juvenile obesity. Relations to body fat depots and insulin concentrations. Am J Physiol. 1996;271(3):E626-E30.

13. Fleisch AF, Agarwal N, Roberts MD, Han JC, Theim KR, Vexler A, et al. Influence of serum leptin on weight and body fat growth in children at high risk for adult obesity. J Clin Endocrinol Metab. 2007;92(3):948-54.

14. De Almeida C, Del Ciampo L, Ricco R, Crott G. Crescimento físico. In: Ricco R, Del Ciampo L, De Almeida C, editores. Puericultura princípios e práticas. 2a ed. São Paulo: Editora Atheneu: 2008. p.43-54.

15. WHO Working Group On Infant Growth. An evaluation of infant growth: the use and interpretation of anthropometry in infants. Bull WHO. 1995;73(2):165-74.

16. Slinger JD, Van BE, Keizer H, Rump P, Hornstra G, Kuipers H. Insulin resistance, physical fitness, body composition and leptin concentration in 7-8 year-old children. J Sci Med Sport 2008;11(2):132-8.

17. Butte NF, Cai G, Cole SA, Wilson TA, Fisher JO, Zakeri IF, et al. Metabolic and behavioral predictors of weight gain in Hispanic children: the Viva la Familia Study. Am J Clin Nutr. 2007;85(6):1478-85.

18. Ahmed ML, Ong KKL, Morrell DJ, Cox L, Drayer N, Perry L, et al. Longitudinal study of leptin concentrations during puberty: sex differences and relationship to changes in body composition. J Clin Endocrinol Metab 1999;84(3):899-905. 
19. Wallace AM, McMahon AD, Packard CJ, Kelly A, Shepherd J, Gaw A, et al. Plasma Leptin and the Risk of Cardiovascular Disease in the West of Scotland Coronary Prevention Study (WOSCOPS). Circulation. 2001;104(25):3052-6.

20. Romero-Corral A, Sierra-Johnson J, Lopez-Jimenez F, Thomas RJ, Singh P, Hoffmann $M$, et al. Relationships between leptin and $C$-reactive protein with cardiovascular disease in the adult general population. Natl Clin Pract Cardiovasc Med. 2008;5(7):418-25.

21. Poveda E, Callas NE, Baracaldo CM, Castillo C, Hernandez P. Leptin levels in school age children associated with anthropometric measurements and lipid profiles. Biomedica. 2007;27(4):505-14.
22. Brandao CM, Lombardi MT, Nishida SK, Hauache OM, Vieira JG. Serum leptin concentration during puberty in healthy nonobese adolescents. Braz J Med Biol Res. 2003;36(10):1293-6.

Artigo recebido: 12/09/08

Aceito para publicação: 16/02/09 